\title{
Editorial
}

\section{Identification of Incipient Damage Using High-Frequency Vibrational Responses}

\author{
Maosen Cao, ${ }^{1}$ Wiesław Ostachowicz, ${ }^{2}$ Gang Li, ${ }^{3}$ Wei Xu, ${ }^{1,4}$ and Mohammad Reza Ashoory \\ ${ }^{1}$ Hohai University, Nanjing 210098, China \\ ${ }^{2}$ Institute of Fluid-Flow Machinery, 80-231 Gdańsk, Poland \\ ${ }^{3}$ Dalian University of Technology, Dalian 116024, China \\ ${ }^{4}$ University of Maryland, Baltimore County, MD 21250, USA \\ ${ }^{5}$ University of Semnan, Semnan 35131-19111, Iran \\ Correspondence should be addressed to Maosen Cao; mmcao@imp.gda.pl
}

Received 11 August 2015; Accepted 11 August 2015

Copyright (C) 2015 Maosen Cao et al. This is an open access article distributed under the Creative Commons Attribution License, which permits unrestricted use, distribution, and reproduction in any medium, provided the original work is properly cited.

Damage identification relying on low-frequency vibrational responses, usually lower than 4th order natural frequencies or so, has dominated the area of vibration-based structural damage diagnosis over recent decades, primarily owing to the limited capacity of traditional apparatus in vibration measurement. In this respect, it is commonly acknowledged that low-frequency vibrational responses are insensitive to small damage; moreover, small damage is more easily accommodated by higher-frequency vibrational responses. This recognition has motivated the development of damage identification methods based on higher-frequency vibrational responses. Currently, new advanced instrumentation typified by the scanning laser vibrometer (SLV) has made it possible to measure high-frequency vibrational responses of a structure precisely and accurately, providing the opportunity to develop damage identification methods using highfrequency (here beyond 4th order) vibrational responses. These high-frequency methods hold promise for solving the critical problem: detection of incipient damage, most crucial for ensuring structural safety. Nevertheless, unlike methods using low-frequency vibrational responses, damage identification relying on high-frequency vibrational responses poses a series of new theoretical and numerical issues that need to be clarified. With this motivation, we have collected papers on this topic in this special issue on identification of incipient damage using high-frequency vibrational responses.

This special issue contains six original research papers that concern various aspects of damage identification relying on high-frequency vibrational responses, including effects of contraction joints on vibrational characteristics of arch dams, diagnosis of bearing defects, estimation of modal properties of low-rise buildings, reliability analysis of damaged beam spectral element, identification of structural damage in bridges using high-frequency vibrational responses, and safety evaluation index of blast vibration.

Maosen Cao

Wiesław Ostachowicz

Gang Li

Wei Xu

Mohammad Reza Ashoory 

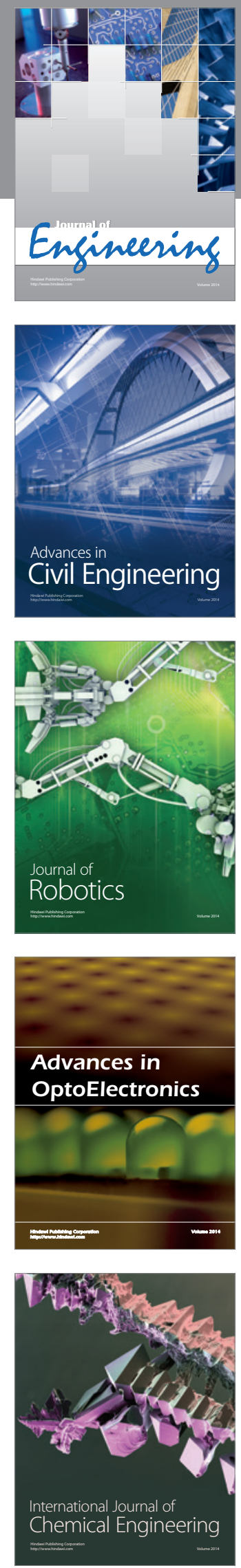

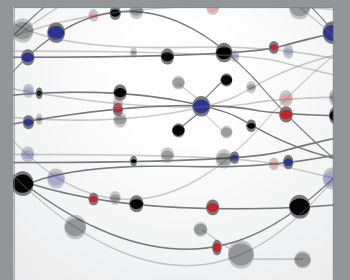

The Scientific World Journal
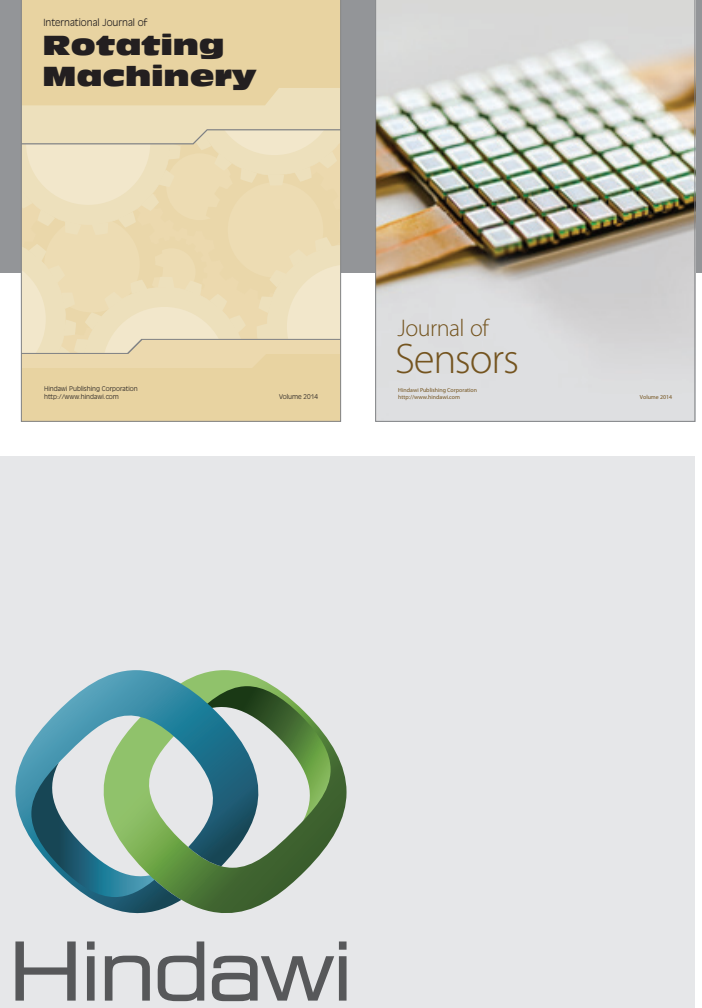

Submit your manuscripts at http://www.hindawi.com
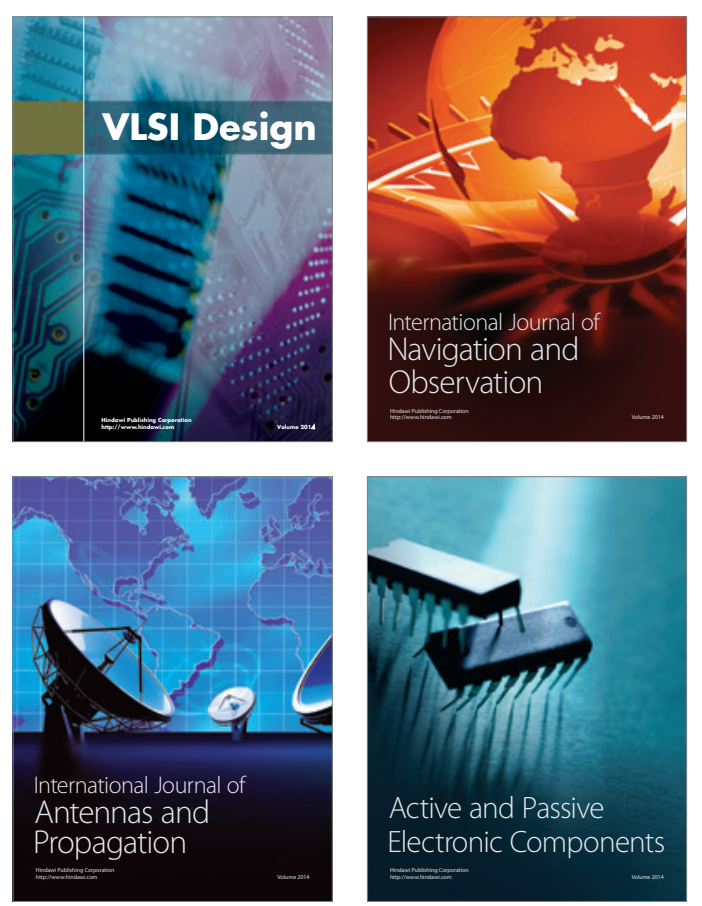
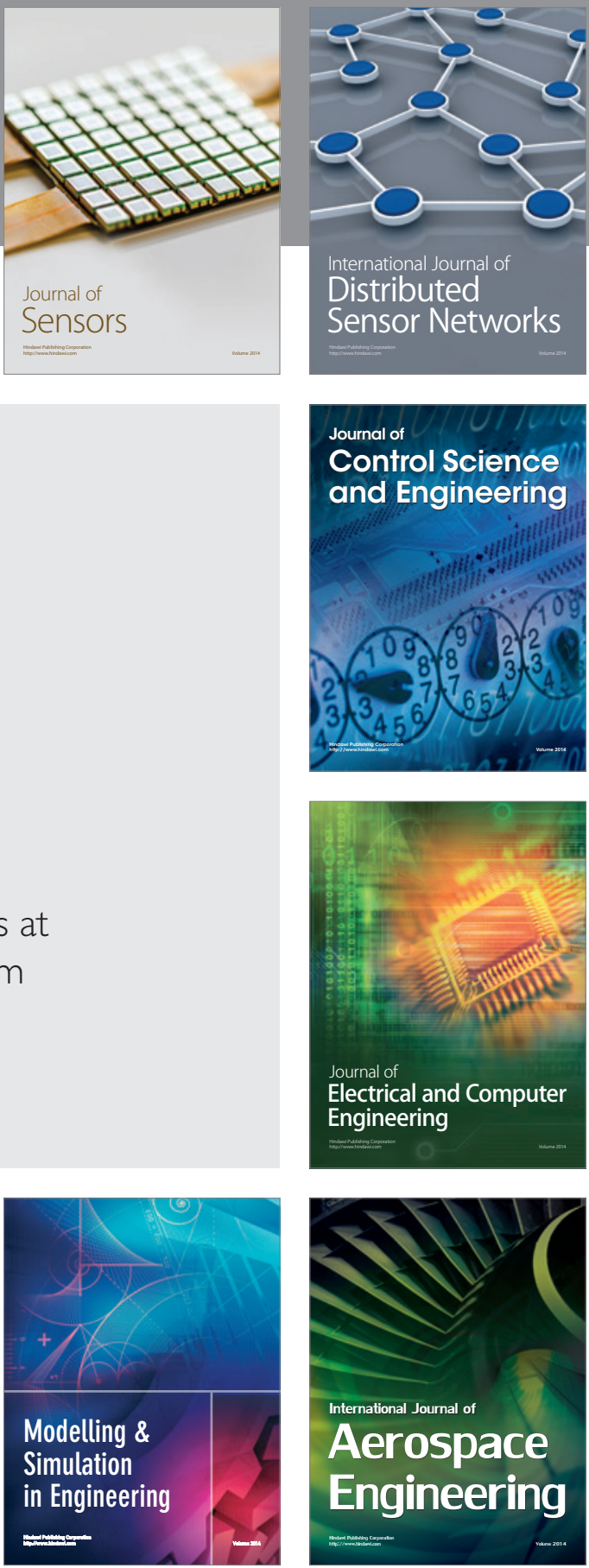

Journal of

Control Science

and Engineering
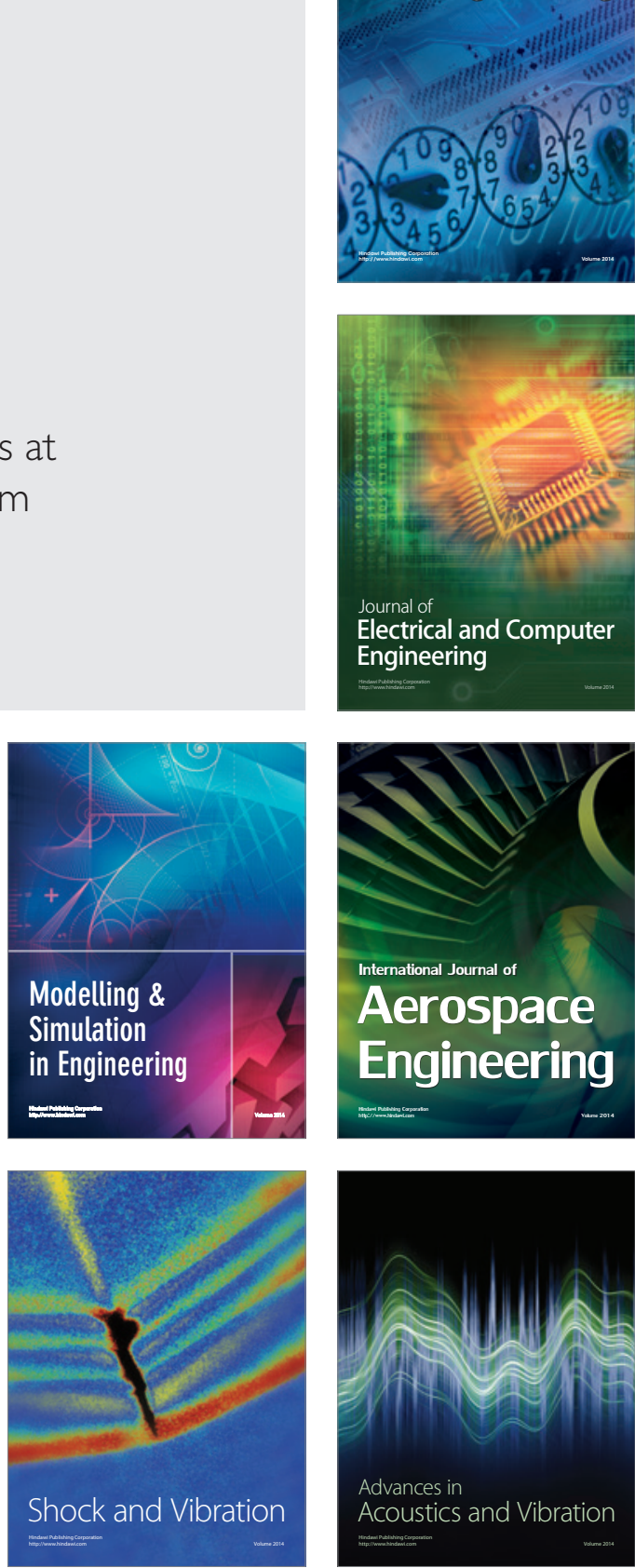